\title{
MAGNETIC FIELDS IN INTERSTELLAR MASERS
}

\author{
J. M. MORAN \\ Harvard-Smithsonian Center for Astrophysics \\ 60 Garden Street \\ Cambridge, MA 02138 USA
}

\section{Introduction and Summary}

Zeeman splitting in the spectra of molecular masers that arise in the envelopes of newly formed stars (called interstellar masers) offers a method of estimating the magnetic field in these dense regions $\left(10^{6}-10^{11} \mathrm{~cm}^{-3}\right)$. The magnetic field strengths in regions of $\mathrm{OH}$ and $\mathrm{H}_{2} \mathrm{O}$ masers scale as the square root of the density when compared to the density and field of the ambient interstellar medium. The direction of the field in $\mathrm{OH}$ masers may help trace the large scale structure of the magnetic field in the Galactic spiral arms.

\section{OH Masers}

OH masers are usually associated with ultracompact HII regions, and they are probably situated in the neutral molecular material outside the ionization boundary. The spectra of $\mathrm{OH}$ masers have many features that are often highly polarized, have widths of $\sim 0.1-1 \mathrm{~km} \mathrm{~s}^{-1}$, and are spread over a range of $2-10 \mathrm{~km} \mathrm{~s}^{-1}$. VLBI observations show that the emission spots associated with the features have sizes $\sim 10^{14} \mathrm{~cm}$ and are spread over $\sim 10^{17} \mathrm{~cm}$ (see Reid and Moran 1988). The discovery of circular polarization in $\mathrm{OH}$ masers (Rogers and Barrett 1966; Davies et al. 1966) was interpreted as evidence of the Zeeman splitting in magnetic fields of $\sim 5 \mathrm{mG}$. However, it is important to realize that although the fields are large enough to separate the Zeeman components in frequency, no complete Zeeman pattern has ever been detected in spite of extensive observations (e.g., Garcia-Barreto et al. 1988). Complex radiative transfer effects in the moving envelopes of the molecular cloud, including effects of velocity and magnetic field gradients and Faraday depolarization, are probably responsible for this situation.

The most convincing evidence for the Zeeman effect comes from interferometric measurements where pairs of oppositely circularly polarized features are seen to emanate from the same position. With interferometry, it is possible in principle to determine the distribution of magnetic field strength over the source. The most extensively investigated source is $\mathrm{W} 3(\mathrm{OH})$. Interferometric maps at three different transitions have allowed the identification of at least 16 Zeeman pairs, whose properties are summarized in the table. At $1665 \mathrm{MHz}$, some of the Zeeman pairs are elliptically polarized, and estimates were made of the position angle of the magnetic field. Note that the $1720 \mathrm{MHz}$ spectra has only a single pair of features, whereas the Zeeman pattern has six $\sigma$ components.

Zeeman splitting has been detected in many $(\sim 20) \mathrm{OH}$ masers. A striking characteristic of these measurements, first noted by Davies (1974), is that most of the magnetic fields in the first and second galactic quadrants point away from the 
Selected Interferometric Observations of W3(OH)

\begin{tabular}{lccccl}
\hline $\begin{array}{l}\text { Transition } \\
\text { Frequency } \\
(\mathrm{MHz})\end{array}$ & $\begin{array}{c}\text { Zeeman } \\
\text { Splitting } \\
\left(\mathrm{km} \mathrm{s}^{-1} \mathrm{mG}^{-1}\right)\end{array}$ & $\begin{array}{c}\text { No. of } \\
\text { Features }\end{array}$ & $\begin{array}{c}\text { No. of } \\
\text { Zeeman } \\
\text { Pairs }\end{array}$ & $\begin{array}{c}\text { Field } \\
\text { Strength } \\
(\mathrm{mG})\end{array}$ & Reference \\
\hline 1665 & 0.60 & 81 & 5 & $5-7$ & Garcia-Barreto et al. 1988 \\
1720 & 0.11 & 2 & 1 & 6 & Lo et al. 1975 \\
6035 & 0.06 & 22 & 10 & $2-9$ & Moran et al. 1978 \\
\hline
\end{tabular}

sun, while those in the third and fourth quadrants point towards the sun. This result suggests that the $\mathrm{OH}$ masers trace an axially symmetric Galactic field. The magnetic field orientation appears to be preserved over a range of densities from $10^{-1} \mathrm{~cm}^{-3}$ in the ambient interstellar medium to $10^{7} \mathrm{~cm}^{-3}$.

\section{Water Vapor Masers}

Water vapor masers are thought to be associated with an earlier stage of star formation than $\mathrm{OH}$ masers principally because they are rarely associated with detectable HII regions. However, in most cases where the proper motions of the maser spots have been measured with VLBI, there is an outflow from a single origin. The velocity spread in $\mathrm{H}_{2} \mathrm{O}$ maser emission is frequently over $100 \mathrm{~km} \mathrm{~s}^{-1}$. The density of maser regions is usually taken to be $\sim 10^{11} \mathrm{~cm}^{-3}$ in order to explain the large luminosity of these masers but stay below the thermalization density.

An indirect estimate of the magnetic field strength was made by Garay et al. (1989) who noted an inverse correlation between the line strength and percentage of linear polarization of the Orion KL maser. According to Goldreich et al. (1973), linear polarization is quenched when the microwave rate in the masing transition exceeds the Zeeman splitting. The microwave rate can be estimated if spherical geometry is assumed and gives an estimate of the magnetic field strength of $30 \mathrm{mG}$. Direct measurements of the unresolved Zeeman effect have been made by Fiebig and Güsten (1989) who observed fractional polarizations of $10^{-4}-10^{-3}$ in several masers and inferred longitudinal magnetic magnetic fields of typically $50 \mathrm{mG}$. These measurements require extraordinary care and attention to instrumental effects. At Haystack Observatory, Barvainis, Haschick, and Moran have begun a program to confirm and extend these results.

\section{References}

Davies, R. D. 1974, in Galactic Radio Astronomy, eds. F. J. Kerr and S. C. Simonson (Dordrecht: Reidel), p. 275.

Davies, R.D., deJager, G., and Verschuur, G. 1966, Nature, 209, 974.

Fiebig, D., and Güsten, R. 1989, Astr. Ap., 214, 333.

Garay, G., Moran, J. M., and Haschick, A. D. 1989, Ap. J., 338, 244.

Garcia-Barreto, J. A., et al. 1988, Ap. J., 326, 954.

Goldreich, P., Keeley, D. A., and Kwan, J. 1973, Ap. J., 182, 55.

Lo, K. Y., et al. 1975, Ap. J., 202, 650.

Moran, J. M. et al. 1978, Ap. J. (Letters), 224, L67.

Reid, M.J., and Moran, J.M. 1988, in Galactic and Extragalactic Radio Astronomy, eds. G. L. Verschuur and K. I. Kellermann (Berlin: Springer-Verlag), p. 225.

Rogers, A. E. E., and Barrett, A. H. 1966, Nature, 210, 188. 
MOUSCHOVIAS: If the total mass of the cloud in which an $\mathrm{H}_{2} \mathrm{O}$ maser is found is $>1 \mathrm{M}_{\odot}$, then I can explain the mGauss fields you observe with my new model for collapsing clouds with ambipolar diffusion.

MORAN: The mass of an $\mathrm{H}_{2} \mathrm{O}$ maser cloudlet is only about $10^{-6} \mathrm{M}_{\odot}$ (e.g. density of $10^{11} \mathrm{~cm}^{-3}$ and a radius of $10^{13} \mathrm{~cm}$ ). However, the total mass of the masing cloud is probably on the order of $1 \mathrm{M}_{\odot}$ or more (e.g. an average density of $10^{6} \mathrm{~cm}^{-3}$ and a radius of $5 \times 10^{16} \mathrm{~cm}$ ). The masing cloudlets are probably condensations in a stellar wind.

HEILES: How can the signs of the $\mathrm{OH}$ maser magnetic fields correlate with the Galactic quadrant when $\mathrm{OH}$ masers are located over a wide range of galactic radius and the Faraday rotation data indicate field reversals? I propose an explanation: $\mathrm{OH}$ masers are located in spiral arms. Thus the field direction in arms must always be the same. The reversals we see must occur in the interarm regions. So we must have all spiral arms with the field pointing inward, and at least some interarm regions with the field pointing outward. Relating this to the observations of external galaxies discussed yesterday by the Bonn group, many galaxies exhibit neither an axisymmetric nor a bisymmetric field pattern, but rather have no simple structure. Perhaps the structure in our Galaxy is an alternative for these external galaxies. 